\title{
The Cytoskeleton Of Toxoplasma Gondii
}

\author{
${ }^{*}$ K. Hu, ${ }^{* *}$ D. S. Roos and *J. M. Murray \\ *Dept. of Cell \& Developmental Biology, University of Pennsylvania, Philadelphia PA 19104 \\ **Dept. of Biology, University of Pennsylvania, Philadelphia PA 19104
}

Toxoplasma gondii is an intracellular parasite and human pathogen. Twenty-two subpellicular microtubules define its cell shape. Fourier analysis of images of isolated, frozen-hydrated subpellicular microtubules demonstrates a distinctive $32 \mathrm{~nm}$ periodicity along the length of the microtubules [1]. A $32 \mathrm{~nm}$ longitudinal repeat is also observed in double rows of intramembranous particles overlying the subpellicular microtubules. The parasite's cytoskeleton also includes an intricate apical structure consisting of the conoid, two intra-conoid microtubules and two polar rings [2]. YFP- $\alpha$-tubulin labels all the tubulin containing structures in $T$ gondii, including subpellicular microtubules, centrioles and spindles, and the apical end of the parasite in the region of the conoid, a 380nm diameter motile organelle consisting of fibers wound into a spiral like a compressed spring. The conoid undergoes a $\mathrm{Ca}^{2+}$ dependent translation of $\sim 0.5 \mu \mathrm{m}$ relative to the lower polar ring [3], causing it to extend at the apical end of the parasite. We observed a second form of motion correlated with extension, an increase in pitch of the conoid fibers. FRAP analysis of $T$. gondii expressing YFP- $\alpha$-tubulin reveals that the conoid fibers are assembled by rapid incorporation of tubulin subunits during early but not late stages of cell division. Deconvolution microscopy and quantitative fluorescence measurements revealed the amount of YFP fluorescence per unit microtubule length [4], and applying these calculations to the intensely labeled apical spot indicated a tubulin content equivalent to $5.5 \mu \mathrm{m}$ of microtubule. The apical elements under study are $\sim 0.3 \mu \mathrm{m}$ long by $0.4 \mu \mathrm{m}$ wide, too small to be well resolved by light microscopy. In negatively stained [1] or in frozen hydrated cryo-EM images, the conoid is marked by a prominent basket-weave pattern, created by superimposition of the 14 conoid fibers on the front and back sides of the organelle. Longitudinal striations with a spacing of $5 \mathrm{~nm}$ can sometimes be seen along the length of each fiber. Each 430nm long fiber completes approximately one-half turn around the conoid as it spirals in a left-handed helix from apex to base. Fourier analysis of images from frozen hydrated samples reveals a rich set of periodic features. Summing the power spectra from several conoid fibers, clear $8 \mathrm{~nm}$ and $4 \mathrm{~nm}$ layer lines are visible. Weaker layer lines are also visible in the power spectra from some conoid fibers, suggesting that their true repeat length is $96 \mathrm{~nm}$. The end-on view of tannic-acid stained conoid fibers shows clear protofilament structure, indicating that tubulin subunits are arranged in columns parallel to the fiber axis, just as in microtubules. Unlike microtubules however, the protofilaments are not arranged as a closed tube, but instead form ribbons of 9 protofilaments folded into a "comma" shape. The profile of these fibers differs radically from the profiles of intra-conoid and subpellicular microtubules in the same sections, lacking circular symmetry, and thus are not derived from ordinary microtubules simply through loss of a few protofilaments. We conclude that the conoid fibers represent a previously unknown arrangement of tubulin.

\section{References}

1. $\quad$ N.S. Morrissette et al.. J. Cell Sci. 110 (1997) 35.

2. $\quad$ B.A. Nichols M.L. Chiappino. J. Protozool. 34 (1987) 217.

$3 . \quad$ R. Mondragon and E. Frixione. J. Eukaryotic Microbiol. 43 (1996) 120.

4. J.R. Swedlow et al. PNAS 99 (2002) 2014. 


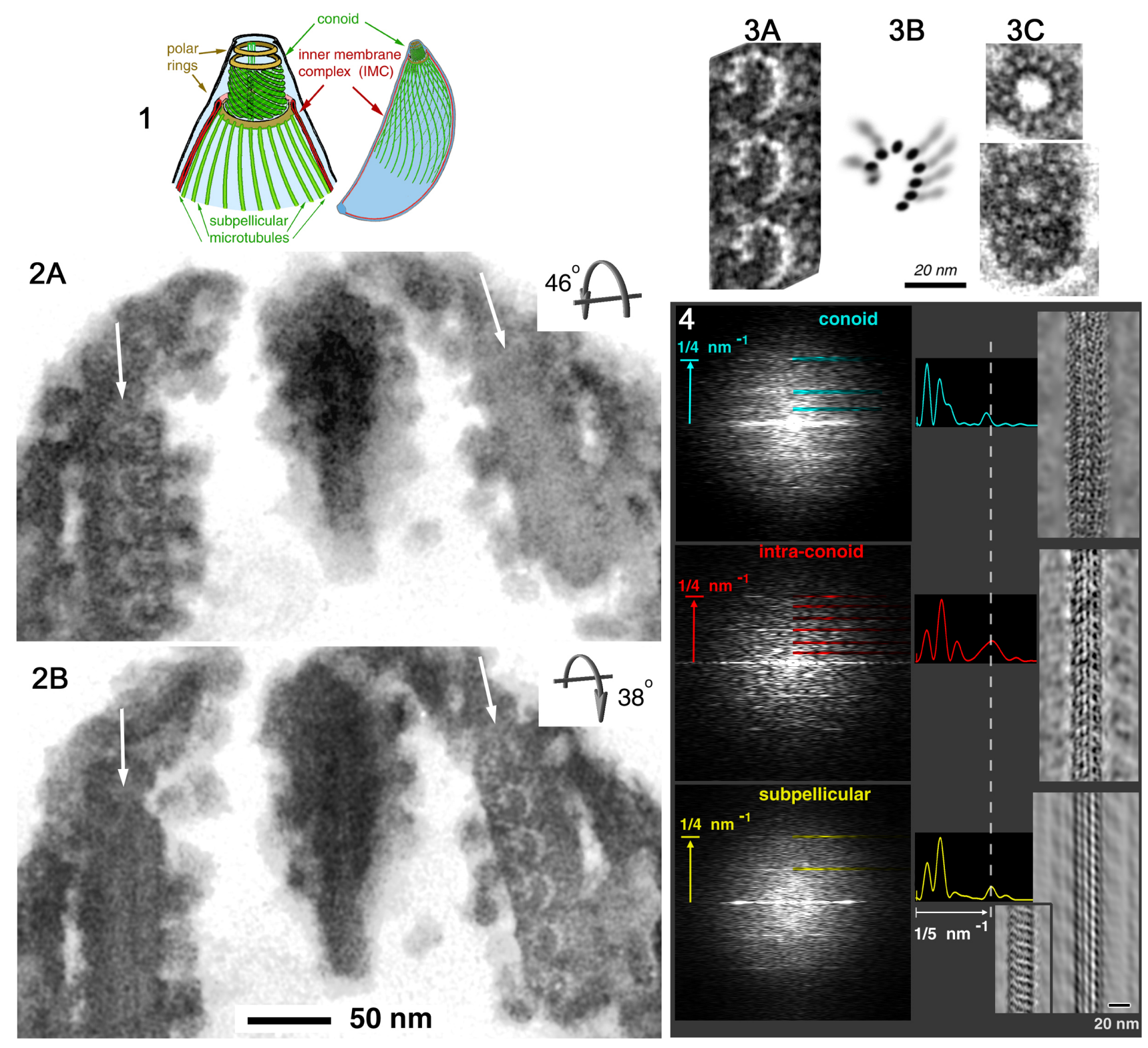

FIG. 1 Drawing of the cytoskeleton of Toxoplasma gondii.

FIG 2A. Thin sagittal section of the conoid tilted $46^{\circ}$ top away from observer. Arrows indicate walls of the conoid. $2 \mathrm{~B}$ The same section as in A, tilted $38^{\circ}$ in the opposite direction.

FIG 3A An average formed by superimposing the aligned images of multiple conoid fibers.

3B Drawing of probable protofilaments (dark ellipses) and associated proteins (elongated gray blobs) in A. 3C EM images of singlet (upper) and doublet (lower) microtubules, for comparison FIG. 4. (left column) Power spectra from conoid fibers and MT structures, oriented with fiber axes vertical. Higher intensities along layer lines indicate strong periodic density fluctuations at intervals of $4 \mathrm{~nm}, 8 \mathrm{~nm}$, and larger spacings, most of which index as higher orders of a fundamental $96 \mathrm{~nm}$ repeat. (middle column) Intensity along the right half of the equator of each power spectrum. (right column) Filtered images from $4 \mathrm{~nm}, 8 \mathrm{~nm}$, and brightest of the remaining layer lines in the Fourier transforms. For subpellicular microtubules, two different images were filtered to show the two different patterns of periodic density fluctuations commonly observed. 